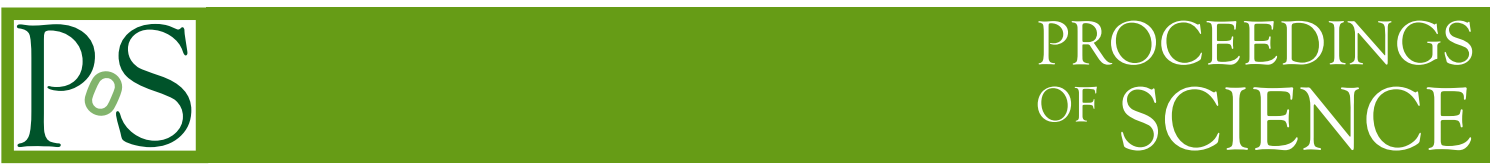

\title{
Calibration and Performance of the CMS Electromagnetic Calorimeter in LHC Run 2
}

\author{
Dmitri Konstantinov ${ }^{a, *}$ \\ ${ }^{a}$ NRC Kurchatov Institute - Institute for High Energy Physics, pl.Pobedi 1, Protvino, Russia \\ E-mail: Dmitri.Konstantinov@cern.ch
}

\begin{abstract}
Many physics analyses using the Compact Muon Solenoid (CMS) detector at the LHC require accurate, high resolution electron and photon energy measurements. The excellent energy resolution is crucial for studies of Higgs boson decays with electromagnetic particles in the final state, as well as searches for very high mass resonances decaying to energetic photons or electrons. The CMS electromagnetic calorimeter (ECAL) is a fundamental instrument for these analyses and its energy resolution is crucial for the Higgs boson mass measurement. Recently the energy response of the calorimeter has been precisely calibrated exploiting the full Run 2 data, aiming at a legacy reprocessing of the data. A dedicated calibration of each detector channel has been performed with physics events exploiting electrons from $\mathrm{W}$ and $\mathrm{Z}$ boson decay, photons from $\pi^{0}$ decays, and from the azimuthally symmetric energy distribution of minimum bias events. The calibration strategies that have been implemented and the excellent performance achieved by the CMS ECAL with the ultimate calibration of Run 2 data, in terms of energy scale stability and energy resolution, are presented.
\end{abstract}

40th International Conference on High Energy physics - ICHEP2020

July 28 - August 6, 2020

Prague, Czech Republic (virtual meeting)

${ }^{1}$ on behalf of the CMS collaboration

*Speaker

(c) Copyright owned by the author(s) under the terms of the Creative Commons 


\section{Introduction}

The Compact Muon Solenoid (CMS) [1] at the CERN Large Hadron Collider (LHC) is a general-purpose detector designed to discover the Standard Model (SM) Higgs boson and search for new physics beyond the SM. The electromagnetic calorimeter (ECAL) [2] is essential for the identification and reconstruction of photons and electrons in CMS. The recent measurement of the mass of the Higgs boson in the diphoton decay channel [3] is an excellent demonstration of precise measurements done with ECAL.

The ECAL is a homogeneous and hermetic calorimeter consisting of 75848 lead tungstate $\left(\mathrm{PbWO}_{4}\right)$ scintillating crystals. The choice of $\mathrm{PbWO}_{4}$ with a radiation length $\mathrm{X}_{0}=0.89 \mathrm{~cm}$ and a Moliere radius $R_{0}=2.19 \mathrm{~cm}$ ensures the compactness of the detector and its radiation hardness in the harsh environment of the LHC. The ECAL is made of a barrel part (EB), covering the pseudorapidity region $|\eta|<1.48$, and two endcaps (EE) extending the coverage up to $|\eta|=3.0$. The schematic ECAL layout is shown in Figure 1.

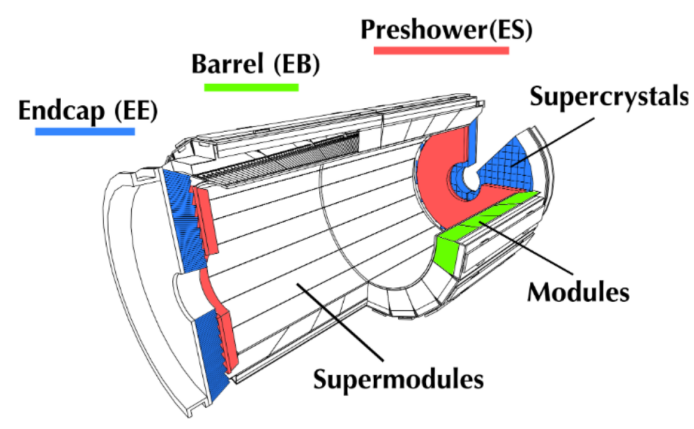

Figure 1: Schematic ECAL layout.

The light from each crystal is detected by an avalanche photodiode (APD) in EB and a vacuum phototriode (VPT) in EE.

\section{Signal amplitude reconstruction}

The signal from the photodetectors is amplified and shaped by a multi-gain preamplifier and then digitized by a 12 bit ADC running at $40 \mathrm{MHz}$, which records ten consecutive samples used to reconstruct the signal amplitude.

Because the LHC colliding bunches are separated by 25 ns at Run 2 and the signal pulses span more than one $25 \mathrm{~ns}$ long time window, the recorded pulse will be the sum of in-time and out-of-time (OOT) pulses. At the beginning of the Run 2, anticipating an increase in the effect of the OOT PU due to the switch from 50 ns to $25 \mathrm{~ns}$ LHC beams, a new algorithm called "multifit" [4] was introduced to minimize the impact of OOT contribution (Figure 2, right). It replaced the Run 1 method [5], where the amplitude was reconstructed as a weighted sum of the ten digitized samples. This algorithm estimates the in-time signal amplitude and up to 9 out-of-time amplitudes by using a template fit. 

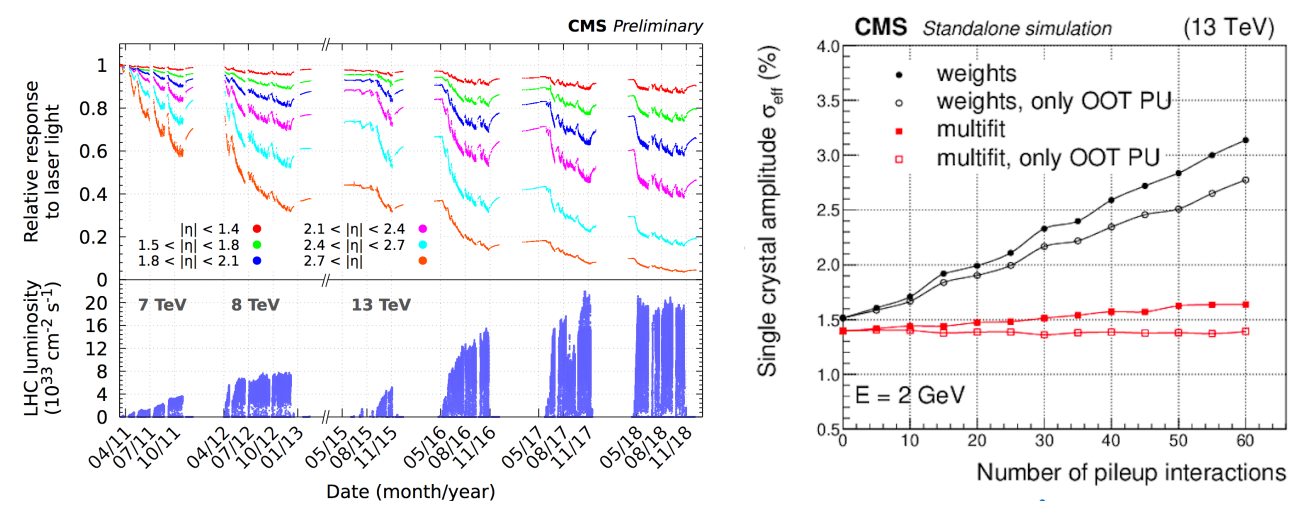

Figure 2: Left: Evolution of the ECAL channel response to laser light versus time since 2011. The channels are subdivided in ranges of $\eta$. The bottom part shows the instantaneous luminosity delivered by the LHC during this period. Right: The 'multifit' reconstruction method is robust to pile-up increase.

\section{Energy reconstruction}

Electrons deposit energy over several ECAL crystals. Clustering algorithms are used to collect the energy deposits in ECAL. The electron or photon energy is estimated as:

$$
E_{e, \gamma}=F_{e, \gamma} G \times \sum_{i}\left(C_{i} \times S_{i}(t) \times A_{i}\right)+E_{E S}
$$

where the index $i$ represents individual crystals within the supercluster, $A_{i}(t)$ is the single channel reconstructed amplitude, $S_{i}(t)$ is the time-dependent crystal response correction, and $C_{i}$ is the channel intercalibration constant. The quantity $G$ is the ADC to $\mathrm{GeV}$ absolute energy scale factor, $E_{E S}$ is the energy deposited in the preshower, and $F_{e, \gamma}$ is the supercluster energy correction.

\section{Crystal response monitoring and intercalibration}

The response of each ECAL channel varies with time. The change is the sum of two effects: crystal transparency variations and changes in photodetector response.

Ionizing radiation creates color centers in the crystal reducing their transparency and therefore reducing their measured response to the deposited energy. The color centers partially anneal with thermal energy such that the loss in transparency depends on the dose rate, which varies with $\eta$, and partial recovery of transparency is observed in the absence of radiation. Changes in crystal transparency and photodetector response are measured (Figure 2, left) and corrected using a dedicated laser monitoring system [6] which injects laser light into each crystal. The changes in response measured by the laser system $\left(R / R_{0}\right)$ are related to changes in the scintillation signal $\left(S / S_{0}\right)$ by means of a power law: $S / S_{0}=\left(R / R_{0}\right)^{\alpha}$.

The monitoring corrections are validated using the stability of the $\pi^{0}$ invariant mass and the stability of the $E / p$ ratio (the isolated $Z / W$ electron energy measured by ECAL divided by the momentum measured by the CMS tracker).

The energy resolution of a calorimeter for high energy electron and photon showers is dominated by a constant term. The main contribution comes from the accuracy of the intercalibration (IC) 

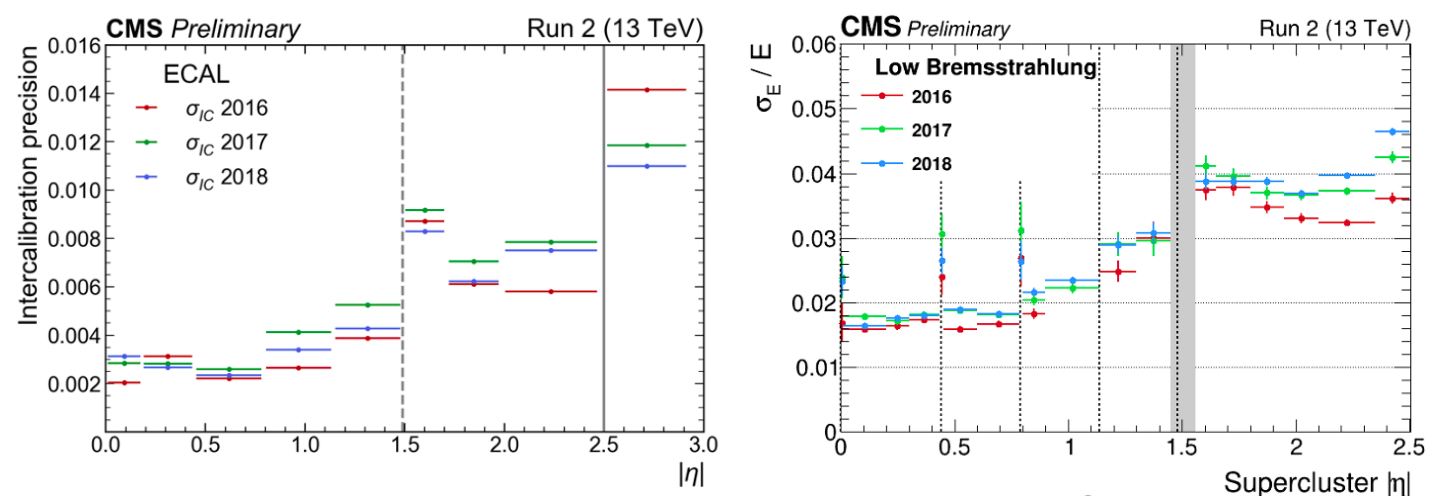

Figure 3: Left: Intercalibration precision computed from IC combination as a function of $\eta$ for different years of data-taking. Right: Relative electron energy resolution for low bremsstrahlung electrons as a function of the $\eta$.

constants which are obtained from LHC collisions data using several independent methods, $\pi^{0} \rightarrow$ $\gamma \gamma, Z \rightarrow e e, E / p$, which exploit the invariant mass of the $\pi^{0}$ and $Z$ particle and the ratio between the independently measured ECAL energy and tracker momentum from $W$ and $Z$ decays. The methods are the same as were used in Run 1 [7]. Intercalibration precision computed from IC combination as a function of $\eta$ for different years of data-taking is shown in Figure 3 (left). The residual miscalibration, shown in Figure 3 (right), is between 0.2 and 1.6\%. Despite the increased radiation damage the same calibration level has been achieved for the entire Run 2.

With the full calibration, ECAL achieved an energy resolution which ranges from 1.5 to $3.5 \%$ in the barrel and from 3.0 to 4.8 in the endcaps for $Z \rightarrow e e$ low bremsstrahlung electrons.

\section{Conclusion}

The CMS electromagnetic calorimeter has demonstrated excellent performance during LHC Run 2. This was achieved by continuous effort in the operation, monitoring and calibration of the calorimeter. Important improvements to the reconstruction and calibration methods have been made to meet the challenges of higher LHC luminosity.

\section{References}

[1] CMS Collaboration, The CMS experiment at the CERN LHC, JINST 3 (2008) .

[2] CMS Collaboration, Technical Design Report CMS-TDR-4-add-1, CERN (2002).

[3] CMS Collaboration, A measurement of the Higgs boson mass in the diphoton decay channel, Physics Letters B $\mathbf{8 0 5}$ (2020) 135425.

[4] CMS Collaboration, Precision electromagnetic calorimetry at the energy frontier: CMS ECAL at LHC Run 2, 1510.02745. 
[5] P. Adzic et al., Reconstruction of the signal amplitude of the CMS electromagnetic calorimeter, Eur. Phys. J. C 46 (2006) 23.

[6] M. Anfreville et al., Laser monitoring system for the CMS lead tungstate crystal calorimeter, Nucl. Instrum. Meth. A 594 (2008) 292.

[7] CMS Collaboration, Energy calibration and resolution of the CMS electromagnetic calorimeter in pp collisions at $s=7 \mathrm{TeV}$, Journal of Instrumentation 8 (2013) p09009. 